\title{
Review of Literature on Iris Recognition Biometrics
}

\author{
GaganpreetKaur $^{\# 1}$, AmritjotKaur ${ }^{* 2}$, \\ ${ }^{1}$ Assistant Professor, Dept. of C.S.E, SGGSWU,Punjab, India \\ 1'gagan1492003@yahoo.com \\ ${ }^{2}$ Student, Dept. of C.S.E, SGGSWU, Punjab, India \\ 2amritjotkaur27@gmail.com
}

Abstract: Biometric system is widelyused and from all the biometric systems iris recognition isbest suited for the purpose: authentication,identification, verifications due to its accuracy and uniqueness.The paper presents the literature review on iris recognition. The body of this paper details the basic steps of iris recognition. The steps are discussed briefly including iris image acquisition, image pre-processing which contains localization, segmentation and normalization, feature extraction and template matching. It also focuses on different literature discussing various techniques of feature extraction.

Keyword-Iris Biometric, Feature extraction

\section{INTRODUCTION}

Biometrics systems are widely used for authentication, identification and verification purposes for any individual [1]-[5].Biometric measurements can be classified as physiological and behavioral characteristics [1] which is unique to a individual.The characteristics used for biometrics include [1],[2] facial features, body odor, features of eye(iris and retina)[2]-[6], thermal emissions, fingerprints, palm prints, voice prints, gesture etc[1].Among these iris recognition is best [3] and accurate method[4] for identification and recognition purpose. Iris recognition is a method of biometric authentication [1]-[4]. It plays an important role in uniquely identifying a person and is based on the uniqueness of iris texture [2]-[6]. This system is one of the mostaccurate systems for identification of individuals. It produces better results in comparison with other biometric systems like face, fingerprint, voice etc [3], [18].Iris recognition system has very low FAR (False Acceptance Rate) [7] and high FRR (False Rejection Rate) [72] compared to other biometric systems. The human iris, as shown in Figure1, has an extraordinary structure and provides abundant texture information [1].

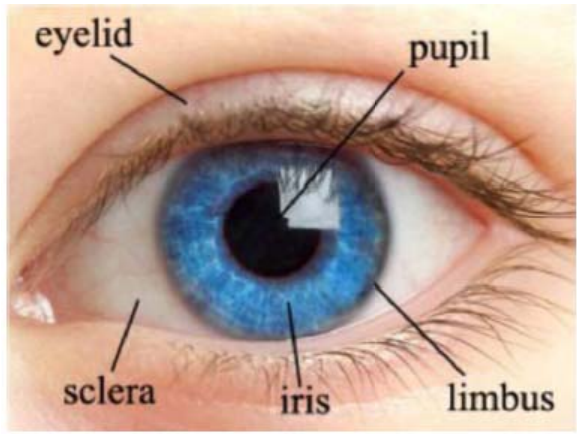

Fig.1. Front view of human eye [63]

\section{MODEL OF IRIS RECOGNITION PROCESS}

A primary iris recognition process includes mainly four steps:

A. Image acquisition

This is the first step in iris recognition. Image acquisition is capturing an eye image from a high resolution camera.It captures the sequence of iris images from subject by cameras and sensors. [4] These images clearly show the entire eye specifically iris and pupil part and then preprocessing operation is applied to enhance the quality of image.

The basic steps of iris recognition system are as shown in Figure 2. 


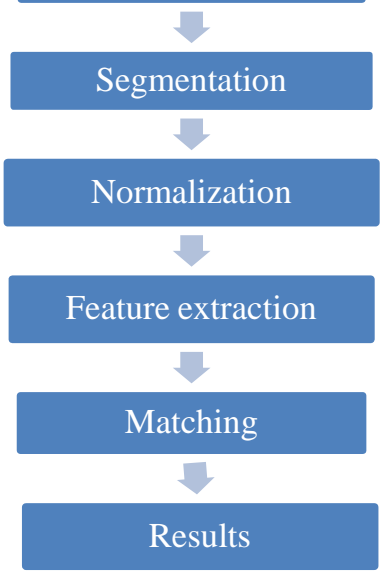

B. Image pre-processing

Fig. 2.Basic steps involved in iris recognition[4]

The pre-processing of eye image is necessary or essential to get the required and accurate input for further processing [5]. Image pre-processing is also necessary to increase the system performance and for the purpose or analysis[71]. It includes Localization, noise removal and normalization.

1) Iris localization: Iris localization is the process to find the iris lower and upper boundary value[5].

This step is detection of the inner boundary and the outer boundary [8] (The inner boundary is the boundary between iris and the pupil and the outer boundary is the boundary between the iris and the sclera).

2) Segmentation: This is the next step. Segmentation if iris depends on the quality of eye images [4].It is a technique required to isolate and exclude the noise and also locates the circular iris region. To isolate the iris portion from the image and alsolocates the circular iris region.It is dependent on quality of eye image[6].

3) Normalization: This is the step followed by segmentation.In this step segmented iris is normalized [4].Normalization is performed for the purpose of achieving more accurate recognition results as some elastic deformation in iris texture will affect the results of iris matching and is necessary to compensate these factors [5].

C. Feature extraction

It involves extraction of most distinct feature of iris. [4] In this step only most discriminating information presented in iris pattern is extracted to provide accurate recognition such that the comparisons can be made. Feature plays a very important role in the area of image processing [36]. Iris feature extraction is used for extracting most distinct feature of an iris image. It contains most of the information of an original iris image. This is one of the major steps in authentication of biometric system. It is used in selection and classification of task. Features are broadly classified as general features and domain specific features.

General features are application independent features like color, texture and shape. It is classified into three categories: Pixel level features, local features and global features.

1) Pixel level features: Features are computed at each pixel.

2) Local features:Features are calculated over the results of image subdivision.

3) Global features: Features are calculated over the whole image.

Domain specific features are also called application dependent features like human face, fingerprint etc.Feature extraction techniques are applied to get different features which are used for classification and recognition of images [36]. Itextracts the most distinct features present in an image. It gives both local and global information of iris. Discriminated iris texture information must be extracted and encoded to have correct comparison between iris templates [15]. Complexity of feature extraction affects the complexity of program and processing speed of iris recognition system [18]. These also used in various image processing applications like character recognition and time consumption also.

Feature extraction can be performed using different mathematical models, image processing techniques and computational tools [37]. These are generally classified in four categories: Feature based, Appearance based, Template based and Part based. 


\section{Matching}

Image matching is one of the most fundamental aspects of image processing[9]. Matching contains comparing iris template for verification.In this step the extracted features of iris are required to match the iris template available indatabase [4]. This step is usually or basically the matching of the feature vectors of an image with feature vectors from any other image [9].

Iris recognition[39] is very accurate and distinct data acquisition due to it its detailed structure and these differences do not change over a long period which make it more specific and unique from other biometric systems.[37] It is a natural authentication method and is based on unique nature of iris.The iris of our 2 eyes is different even for identically appeared twins. It is one of the most reliable methods. It is biometrically based technology which is helpful for identification and verification purposes with individually iris prints [39].It has many applications like it is used in airport security, construction programs and military trainings or applications.It provides unique identity even for those who do not have a passport or identity card. Iris recognition is most powerful identification feature among all other biometric features due to its reliability,accuracy and uniqueness[38].

In the next section detailed literature review for the iris recognition is discussed.

\section{LITERATURE REVIEW}

W.W.Boles et al [31] in 1998 has proposed a novel technique for iris recognition which offers freedom in image capturing as it is translation and size invariant. The proposed algorithm was tested using real images. In this work the iris pattern of single eye was used to construct the representations in the database. The results had shown the correctly classification and recognition of the different iris patterns.Li. Ma et al[32] in 2002 proposed a new method for personal identification based on iris recognition. The new approach is considered of three main components: image pre-processing, feature extraction and classifier design and a set of filters is applied. The proposed new algorithm was effective and also achieved high performance.In 2002, Li Ma et al [1] proposed an algorithm for personal identification based on iris recognition, the steps of which include image pre-processing, feature extraction and classifier design. This algorithm is more reliable, faster and achieves much higher identification rate. It effectively distinguishes different persons by identifying their irises.In 2006, Jun-Boa Li et al[10] proposed a novel matrix norm based on Gaussian kernel for feature extraction of images. Gaussian kernel is widely used in support vector machines and other kernel methods and provides local measure of similarity between vectors [10]. It brings large storage requirements and the large computational efforts for transforming images to vectors. The performance of M-Gaussian kernel is better than conventional Gaussian kernel.S.Poonguzhaliet al [27] in 2006 has performed evaluation of feature extraction for classifying abnormalities in ultrasound liver images using neural network. In this texture features were extracted by different methods and then were to classify the sets of ultrasonic liver images. The neural network classifier is used in this work for evaluation. The Gabor wavelet method among all other methods shown the high recognition rate as it gives best recognition. John Daugmanet al [41] in 2007 has presented advancement in methods of iris recognition and showed more discipline methods for detecting and modeling the iris inner and outer boundaries, Fourier Based methods and statistical inference method for detection and exclusion of eyelashes and also explored a method for normalization.

Hugo Proencaet al [42] in 2007 focused the work on non cooperative iris recognition and proposed an iris classification method. With the implementations it resulted in decrease of number of error rate and improved flexibility.Zhenan Sum et al [43] in 2009 proposed a method using ordinal measure for iris feature representation. It achieved a good trade-off between distinctiveness and robustness. It resulted as powerful tool for complex tasks. Ordinal measures are used for image acquisition. The proposed methods computationally complexity was low and highly efficient.Karen P.GaganpreetKauret al [72] in 2010, has discussed about enhanced iris recognition. The paper presented an novel and automated iris recognition system in which accuracy is increased and computational speed is reduced. Hollingsworth et al [44] in 2011 has defined a new metric called the fragile bit distance. The work is focused on the fusion of fragile bit distance and hamming distance. It is better classifier. With the fusion it reduces the error and showed the improvement. The performance was superior with the fusion of algorithms than the algorithms used individually.In 2012, Dolly Choudharyet al discussed feature extraction methods for iris recognition [4]. These include image acquisition, segmentation, normalization, feature extraction, matching. The feature extraction methods discussed include Corner detection based iris encoding, Feature extraction using Hear Wavelet, feature extraction using Gabor filter, Statistical pattern recognition and Multichannel Gabor filter.

In 2013, Rodrigo D.C Silva et al discussed images extracted from a 3D industrial sensor using seven feature extractors and 3 classifiers. They reported a comparative study between seven invariant moments (Hub, Zernike, Legendre, Fourier-Mellon, Tschebichef, Basel Fourier and Gaussian- Hermit) and independent component analysis as feature descriptors of images from different databases. The three classifiers were k-NN classifier, neural network based classifier and support vector machine. The feature extraction step revealed that Zernike moments and Castor were good candidates for feature description with slight advantage of Castor when k-NN is 
adopted as classifier [3]. The study of classifier revealed the superiority of the SVM when the Zernike moments are used as a feature description.Jan Barabaset al [33] in 2013 developed the software which is replacement of available system. The proposed algorithms may also be used in areas of image manipulation and feature extraction. The new work was more appropriate than the lasting one.ShuxiangGuoet al [34] in 2013 proposed a novel feature extraction method for Semg signals using image processing. The work is focused on feature extraction of Semg signals by calculating geometric feature of these signals. It used PCM (pixel count method) for feature extraction and ASM (Angular second moment) for textural features. The proposed method is superior and processing time was very short from other methods.

Chun-Wei Tan et al [35] in 2014 worked on efficient and accurate at-a-distance iris recognition using geometric key based iris encoding. Such iris encoding is computationally more efficient. The proposed approach used both visible and NIR imaging. The features were extracted both locally and globally. The proposed algorithm was improvement from others when compared.Gaurav Kumar et al [36] in 2014 reviewed detail in feature extraction in image processing systems. Firstly pre-processing techniques were applied and then feature extraction techniques are applied for further classification and recognition. The work is focused on feature extraction techniques for character recognition applications.Siliviobarraet al [26] in 2014 has used a comprehensive method on mobile devices for iris authentication. The proposed approach performed well on iris database. The implementation of iris recognition was feasible and accuracy was improved.Vivekpaliet al[37] in 2014 reported an extensive survey on different types of feature extraction techniques. The reported techniques are classified into four types which can also be used in face recognition problem. It provided comparative study on these techniques.In 2014, Side Ali et al introduced hardware architecture for difference of Gaussian calculation in image feature extraction. Image matching techniques consist of features extraction and their matching with other features. They constructed hardware architecture for steps involved in the process. This approach resulted in much faster FPS as compared to others. The results were shown to be better than previous implementations in terms of required hardware resources [9].

Estefan Ortiz et al [2] in 2015 incorporated pupil dilation information into selection of images for enrolment using empirical distribution of dilation ratios for each iris to be enrolled which shows improved accuracy in iris recognition [2]. They used a quantile-based approach to dilation-aware enrolment which finds quantile points for each subject's distribution of dilation values. In this strategy, they used a commercial iris matcher (VeriEye) and found incorporating pupil dilation information into the selection of images for enrolment improves the accuracy of iris matching. The dilation-aware enrolment has measurable performance increases over the baseline random enrolment.In 2015,Albadarneh. A et al [71], has reviewed and evaluated four iris pattern recognition features which includes histogram of oriented gradients, gray level co- occurence matrix and combined Gabor and discrete cosine transform. The results of proposed work shows that GLCM was most effective technique and also give largest recognition accuracy among others.In 2016, Silencer S et al used hybrid methods of feature extraction and matched them by hybrid classifiers for iris recognition system[5]. They discussed the feature extraction using Haar transform, PCA, Block sum algorithm with hybrid algorithm. These transforms were applied on iris images for finding the recognition rate and accuracy. The hybrid classifiers used in this study were, ANN and FAR/FRR. The experimental technique produced good performance on CASIA VI iris database. This proposed algorithm provides better accuracy and recognition rate than comparative algorithms.

In 2016, Ajay Kumar et al used cross spectral matching for more accurate iris recognition. Matching is done by two different domains; sensor specific and wavelength specific respectively[6].It developed domain adaption framework which introduced a new algorithm Markov random fields (MRF) models to improve cross domain iris recognition. The proposed framework was based on naïve Bayes nearest neighbor classification. It presented three publicly available databases; PolyU cross spectral iris image database, IIITD CLI and UND database. The proposed method for cross spectral iris recognition was capable of benefitting from the final match score distribution of both the domains. The performance improvement for the above proposed method was higher as compared to performance of cross sensor iris recognition.In 2016, Mengzhe Li et al proposed an improved algorithm based on color feature extraction for image retrieval [7]. There were two main types of algorithms based on color features: Global algorithm and Local color algorithm and so on. The proposed algorithm considers the global and local color information, combining the fuzzy color histogram and block color histogram. It also compared different histograms with improved algorithm. This was an effective method for image retrieval and also saves the storage space in the system. This improved algorithm has a better performance than other algorithms.Harini R et al [28] in 2016 proposed a method for DR detection by using FCM(Fuzzy C-means) clustering and morphological image processing. It used SVM classifier. The proposed method achieved more accuracy and sensitivity. 
In 2016, Tossy Thomas et al, has proposed a more accurate method called RANSAC (Random Sample Consensus). It located iris boundaries more accurately than the methods based on Hough transform. For iris normalization Daugman's rubber sheet model was used along with WVU database. Ellipse fitting through RANSAC provided a better result in iris localization compared to Hough transformed result and the performance of this system was better than Daugman's iris recognition system [8].While,AlokeDattaet al[11] in 2016 used partitioned maximum margin criteria (MMC) for supervised feature extraction of hyper spectral images. The main two approaches were feature selection and feature extraction. They proposed a feature extraction technique to reduce the Hughes phenomenon and computational complexity of the system. The proposed method was supervised in nature. The proposed method effectiveness is signified both qualitatively and quantitatively with five state-of- the-art techniques. The proposed method gave improvement, more consistency and better performance as compared to other methods [11].

Abhineet Kumar et al[18] in 2016 gave comprehension review of different iris feature techniques used in iris recognition system.This includes the latest development in terms of accuracy and complexity of iris recognition system.[18] The various methodologies of iris feature extraction were discussed such as Gabor filter, wavelet transform, Haar Wavelet, Gabor Wavelet, Dual tree complex wavelet transform, contour let transform, wavelet packet and fusion of 2D Gabor and 1D log-Gabor. It helped to increase the accuracy and reduce the complexity in iris recognition system. Qi Zhang et al [23] in 2016 explored complementary features for iris recognition on mobile devices. The features are explored to improve the accuracy. In this the om features and pairwise features are explored individually and then fused at one level. The fusion results demonstrated that these features are highly complementary and effective. Their algorithms used for fusion are faster than all others.Kiichifukumaet al [24] in 2016 had studied on feature extraction and disease stage classification for giloma pathology images. In this nucleus segmentation method and feature descriptors were discussed.With the implementations it improved the accuracy of nuclei segmentations.

Yosefinafinsensiaritiet al [25] in 2016 has used feature extraction for lesion margin characteristic classification from ct scan lungs image. This scan provided more opportunity for survival. Using computer based digital image processing improved the accuracy inresults of the ct scan images. In this the work is done in stages like segmentation process and feature extraction methods with different number of features and classification process. It improved the accuracy of classification feature process.

TABLE I shows the iris recognition papers focusing on techniques used for feature extraction.

TABLE I. Techniques Used for Feature Extraction in Iris Recognition System from Year 1998-2017.

\begin{tabular}{|l|l|l|l|}
\hline Year & Methodology/ Technique & Stage & Ref. \\
\hline 1998 & Wavelet transform zero crossing & Extraction of unique features & 48 \\
\hline 2002 & Circular symmetric filter & $\begin{array}{l}\text { Capturing image information in specific } \\
\text { frequency band }\end{array}$ & 49 \\
\hline 2002 & ICA (Independent Component analysis) & Extract iris texture feature & 50 \\
\hline 2002 & Multichannel Gabor filtering & Image processing for FE for texture analysis & 4 \\
\hline 2003 & Statistical Pattern recognition & FE using FFT & 31 \\
\hline 2004 & Gabor filter & Quantization feature extraction & 12 \\
\hline 2009 & Contour let transform & Matching feature extraction & 14 \\
\hline 2010 & Cumulative sum based analysis method & $\begin{array}{l}\text { Analyzes grey value patterns in iris images } \\
\text { and extracts iris features }\end{array}$ & 72 \\
\hline 2012 & LPCC & Extract features in speech signal & 52 \\
\hline 2012 & Corner detection based iris encoding & Texture, iris localization & 36 \\
\hline 2012 & Gabor wavelet & Edge detection & 53 \\
\hline 2012 & Gabor wavelet & Matching feature extraction & 13 \\
\hline 2012 & Gabor filter & Normalization & 43 \\
\hline 2013 & $\begin{array}{l}\text { Fusion of Haar wavelet and 1D log gabor } \\
\text { wavelet }\end{array}$ & $\begin{array}{l}\text { Data analysis in iris region and feature } \\
\text { encoding }\end{array}$ & 54 \\
\hline 2013 & DWT & Wavelet transformation & 55 \\
\hline 2013 & Fusion of 2D Gabor and 1D log Gabor & Extraction process & 17 \\
\hline 2014 & GEFE & Texture classification & 56 \\
\hline 2014 & MLBP(modified local binary pattern) & Iris texture classification & 57 \\
\hline 2014 & $\begin{array}{l}\text { Sobel, canny and prewitt edge detection } \\
\text { methods }\end{array}$ & Image pre processing & 69 \\
\hline
\end{tabular}




\begin{tabular}{|l|l|l|l|}
\hline 2014 & SIFT algorithm & Gaussian difference & 9 \\
\hline 2014 & $\begin{array}{l}\text { Neu-wave network(Combo of haar } \\
\text { wavelet + neural network ) }\end{array}$ & Extraction of significant iris feature & 20 \\
\hline 2014 & $\begin{array}{l}\text { Global iris bits stabilization+ local ZM's } \\
\text { phase based encoding method }\end{array}$ & $\begin{array}{l}\text { Recovery of iris features by } \\
\text { matchingprocess. }\end{array}$ & 58 \\
\hline 2015 & Principle Component Analysis & $\begin{array}{l}\text { Compression of datasets from higher to } \\
\text { lower dimensions in feature extraction }\end{array}$ & 35 \\
\hline 2015 & Sift & Key points based feature extraction & 59 \\
\hline 2015 & Gabor filters bank & Discriminate texture of an image & 60 \\
\hline 2015 & Multi scale morphology algorithm & Extract structural/ textural features & 61 \\
\hline 2015 & Hybrid combo of DCT and Gabor filter & Pattern recognition & 71 \\
\hline 2016 & SURF (speeded up robust features) & Key points detection & 62 \\
\hline 2016 & CLBP & Local texture feature extraction & 63 \\
\hline 2016 & Rubber sheet model & Remapping of iris region & 64 \\
\hline 2016 & LDA(Linear discriminant analysis) & Characterization of 2 or more classes & 65 \\
\hline 2016 & Convolution technique & Face part detection & 66 \\
\hline 2016 & Micro dimensional GEFE & Data analysis & 67 \\
\hline 2016 & $\begin{array}{l}\text { Fusion of OM features and pair-wise } \\
\text { features }\end{array}$ & $\begin{array}{l}\text { Encoding of local iris texture and measure } \\
\text { of co-relation between 2 iris using CNN }\end{array}$ & \\
\hline 2016 & Block Sum plus Haar Transform & Generation of iris code & 19 \\
\hline 2016 & $\begin{array}{l}\text { Partitioning of hyper spectral features and } \\
\text { uses MMC based transformation. }\end{array}$ & Supervised feature extraction & 11 \\
\hline 2016 & $\begin{array}{l}\text { Hybrid Algorithm (combination of block } \\
\text { sum and haar transform) }\end{array}$ & Feature vector and testing & 70 \\
\hline 2017 & Discriminative statistics & Iris classification & \\
\hline & & & \\
\hline
\end{tabular}

\section{REFERENCES}

[1] Ma, Li., Wang. Y, Tan. T., “ Iris Recognition Based On Multichannel Gabor Filtering”, (2002).

[2] Ortiz. E, W. B. Kevin, J. F. Patrick, “Dilation-aware enrolment for iris recognition”, The Institution of Engineering and Technology (2015).

[3] Rodrigo D.C. Silva, George A.P. The, Fatima N. S. de Medeiros, "Geometrical and statistical feature extraction of images for rotation invariant classification systems based on industrial devices”, IEEE (2013).

[4] Choudhary. D, Tiwari . S, Singh. A.K, “A Survey: Feature extraction methods for iris recognition”, IJECCT, Volume 2, Issue 6 (2012).

[5] Gale. A. G, Salankar. S. S, "Evolution of performance analysis of iris recognition system by using hybrid methods of feature extraction and matching by hybrid classifier for iris recognition system”, IEEE (2016).

[6] Ramaiah. N. P, Kumar. Ajay, “Towards more accurate iris recognition using cross-spectral matching”, IEEE (2016).

[7] M. Li, Jianj. X, "An Improved algorithm based on color feature extraction for image retrieval”, IEEE (2016).

[8] Thomas. T , George. A ,Devi. K. P. I, “Effective iris recognition system”, RAEREST (2016).

[9] Bukhari. S.A.A, Iqbal. S, “A Hardware architecture for difference of Gaussian calculation in image feature extraction” , IEEE (2014).

[10] Jun. Li, Chuan. Chu, S. Pan and J.H. Ho, "A Novel matrix norm based Gaussian Kernel for feature extraction of images”, IEEE (2006).

[11] Datta. A, Ghosh. G, Ghosh. A, "Supervised feature extraction of hyper spectral images using partitioned maximum margin criterion", IEEE (2016).

[12] Daugman, “How iris recognition works” IEEE Trans. On Circuits and Systems for video technology Vol.14, No.1(2004).

[13] Gulmire. K, Ganokar. S, “Iris Recognition using Gabor Wavelet”, IJERT,Vol.1 Issue 5, (2012).

[14] Azizi.A, Pourreza. H A' "Efficient iris recognition through improvement of feature extraction and subset selection" ,IJECSI, Volume 2, No 1 (2009).

[15] T. W. Ng ,T. L. Tay, S. W. Khor, "Iris recognition using rapid haar wavelet decomposition", IEEE ,(2010).

[16] Elgamal. M ,Nassar A.B, "An efficient feature extraction method for iris recognition based on wavelet transformation, IJCIT (2013).

[17] Y. Zhang, "A fusion iris extraction based on linear discriminant", IEEE (2013).

[18] Kumar. A, Potnis .A ,Singh . A. P, "Latest development in feature extraction techniques in iris recognition system", IRJET, (2016)

[19] Verma. K. K, Kumar. P, Tomar. A and Srivastava. M, "A Comparative study of image segmentation techniques in digital image processing”, IJEETC ,Vol.1,No.2, (2015).

[20] Moi .S. H, Asmuni. H, Hassan. R,Othman. R. M, "Multimodal biometrics:weighted score level fusion based on non ideal iris and face images", ELSEVIER, (2014).

[21] Nsibi. S .S, Benyahia .A .B, “Region-based image retrieval using a joint scalable Bayesian Segmentation and feature extraction”, IEEE (2016).

[22] Verma. K. K, Kumar. P, Tomar. A and Srivastava. M, “A Comparative study of image segmentation techniques in digital image processing”, IJEETC ,Vol.1,No.2, (2015).

[23] Zhang . Q, Li. H, Sun. Z , He. Z and Tan. T, “Exploring complementary features for iris recognition on mobile devices”, IEEE (2016).

[24] Fukuma. K ,SuryaPrasath. V. B ,Bruce J. Aronow, “A study on feature extraction and disease stage classification for giloma pathology images”, IEEE (2016). 
[25] Riti. Y. F, Nugroho. H. A, Wibirama. S, Budi. W and Choridah. L, "Feature extraction for lesion margin characteristic classification from ct scan lungs image”, IEEE (2016).

[26] Barra. S, Casanova. A, Narducci. F, Stefano. R, “Ubiquitous iris recognition by means of mobile devices”,(2014).

[27] Poonguzhali. S, Ravindran. DR.G, "Performance evaluation of feature extraction methods for classifying abnormalities in ultrasound liver images using neural networks”, IEEE (2016).

[28] Harini. R, Sheela. N, "Feature extraction and classification of retinal images for automated detection of diabetic retinopathy", IEEE (2016).

[29] Omer M. S, Chen. J and Schneider. H, “An Image processing tool for efficient feature extraction in computer aided detection systems”, IEEE (2010).

[30] Chan. W. T, Swee. S. K, "Experimental method to pre-process fuzzy bit planes before low-level feature extraction in thermal images”, IEEE (2015).

[31] Choudhary. D, Tiwari . S, Singh. A.K, “A Survey: Feature extraction methods for iris recognition”, IJECCT, Volume 2, Issue 6 (2012).

[32] Jan . B, Roman. R and Daniela. G, “Image processing and feature extraction of circular objects from biological images”, IEEE (2013).

[33] Guo. S, Guo. C, Pang. M, “A novel feature extraction method for semg signals using image processing”, IEEE (2013).

[34] Gale. A. G, Salankar. S. S, "Evolution of performance analysis of iris recognition system by using hybrid methods of feature extraction and matching by hybrid classifier for iris recognition system", IEEE (2016).

[35] Choudhary. D, Tiwari . S, Singh. A.K, “A Survey: Feature extraction methods for iris recognition”, IJECCT, Volume 2, Issue 6 (2012).

[36] Pali. V, Goswami. S and Bhaiya. L. P, “An extensive survey on feature extraction techniques for facial image processing”, IEEE (2014).

[37] Kaur. N and Juneja. M, “A review on iris recognition”, IEEE (2014).

[38] Navarro .L .A, Dino. M. A, Joson. E, Rommel. A, Roberto. D. C, "Design of alcohol detection system for car users through iris recognition pattern using wavelet transform”, IEEE (2016).

[39] Alsubari. A and Ramteke. R. J, "Extraction of palm print texture feature using combined DWT-DCT and local binary pattern”, IEEE (2016)

[40] Daugman. J, “New method in iris recognition”, IEEE, Vol.37, No 5(2007).

[41] Nsibi. S. S ,Benyahia A.B , "Region-based image retrieval using a joint scalable Bayesian Segmentation and feature extraction”, IEEE (2016).

[42] Choudhary. D, Tiwari . S, Singh. A.K, “A Survey: Feature extraction methods for iris recognition”, IJECCT, Volume 2, Issue 6 (2012).

[43] Karen P. Hollingsworth, Kevin W. Bowyer and Patrick J. Flynn, "Improved iris recognition through fusion of hamming distance and fragile bit distance”, IEEE, Vol.33, No.12 (2011).

[44] Zhuoshi. W, Xianchao. Q, Zhenan Sun and Tan. T, " Counterfeit iris detection based on texture analysis", IEEE, (2008)

[45] Zhaofeng. H, Tan. T, Zhenan. S, Xianchao .Q, "Toward accurate and fast iris segmentation for iris recognition", Vol 31, No 9 , IEEE (2009).

[46] Kumar. S. R, G. R. S, X. Nithiya, X. Sharmi, " A Study on iris recognition system ", Vol. 2, Issue. 7, IJCSMC.

[47] Boles. W . W, Bashash.B, " A human identification technique using images of the iris and wavelet transform" IEEE, (1998).

[48] Li. Ma, Wang. Y, Tan. T," Iris recognition using circular symmetric files" IEEE, (2002).

[49] Huang. Y. P, Luo. S.W , Chen. E.Y,"An efficient iris recognition system" ,IEEE, (2002)

[50] Chu .C.T, Chen . C. H,"A novel iris recognition based on lda and lpcc", IEEE, (2012).

[51] Gulmire. K, Ganorkar. S, " Iris recognition using gabor wavelet", IEEE, (2012).

[52] Sun. Z, Zhang. H, Tan. T, "Iris image classification based on hierarchical visual codebook", IEEE, (2013).

[53] Rai. H, Yadav. A, "Iris recognition using combined support vector machine and hamming distance approach", IEEE, (2013).

[54] Elgama .M .M ,Biqami. N.A, "An efficient feature extraction method for iris recognition based on wavelet transformation", IEEE (2013).

[55] Connor. B. O, Roy. K ,Shelton . J, Dozier. G," Iris recognition using fuzzy level set and gefe" ,IEEE,(2014).

[56] Popplewell. K, Roy. K, Ahmad. F, Shelton. J, "Multispectral iris recognition utilizing Hough transform and modified lbp" , IEEE, (2014).

[57] Tan. C.W, Kumar. A, "Accurate iris recognition at a distance using stabilized iris encoding and zernike moments phase features", IEEE, (2014).

[58] Betancourt .Y. A, Silvente. M. G, "A key points based feature extraction method for iris recognition under variable image quality conditions", ELSEVIER,(2015).

[59] Joshi. A. G, Deshpande .A. S, "Feature extraction of face images using weber's local descriptor for recognition", IJCITE, (2015).

[60] Umer. S, Dhara. B .C, Chanda .B, " Iris recognition using multi scale morphological features", (2015).

[61] Agrawal. A, Samson. S, "A review on feature extraction techniques and general approach for face recognition" IJCAT, Volume 5, issue 3, (2016).

[62] Devi. K ,Gupta. P, Grover. D, Dhindsa . A, "An effective feature extraction approach for iris recognition sytem", Indian journal of Science and Technology, (2016).

[63] Singh. J. P, Varshney. S, "Improving iris recognition performance using morphological bridged canny edge detection and pixel based matching algorithm", IJESC, Volume 6, Isuue no 5, (2016).

[64] Agrawal. A, Samson. S, "A review on feature extraction techniques and general approach for face recognition" IJCAT, Volume 5, issue 3, (2016).

[65] Agrawal. A, Samson. S, "A review on feature extraction techniques and general approach for face recognition" IJCAT, Volume 5, issue 3, (2016).

[66] Shelton . J, Roy. K,"Micro dimensional feature extraction for multispectral iris recognition", IEEE (2016)

[67] Zhang. Q, Haiqing. L, Zhenan. S, Tan. T, "Exploring complementary features for iris recognition on mobile devices" IEEE, (2016).

[68] Nair. S.A .H, Aruna .P ,"Sparse representation fusion of fingerprint, iris and palm print biometric features" ,International Journl of advanced computer research, Volume 4, Isuue no 14, (2014).

[69] Fathy. W. S. A, Ali. H. S, Mahmoud. I. I, "Statistical representation for iris anti spoofing using wavelet based feature extraction and selection algorithm", IEEE, (2017).

[70] Albadarneh. A, Albadarneh. I, Alqatawna. J. F, "Iris recognition system for secure authentication based on texture and shape features", IEEEE, (2015).

[71] Kaur. G, Gindhar. A, Kaur. M, "Enhanced iris recognition system : An integrated approachto person identification", International Journal of Computer Application, Volume 8, No. 1,(2010). 


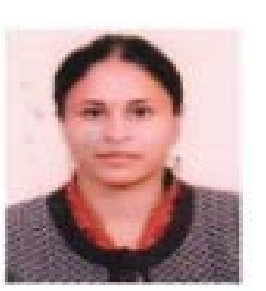

\section{Author Profile}

Gaganpreet Kaur Assistant Professor Deptt.of C.S.E, SGGSWU, F.GS., Punjab She has done B. Tech from Kurukshetra University with Honors and M. Tech from Guru Nanak Dev Engineering College, Ludhiana. She is pursuing Phd from I.K.Gujral Punjab Technical University. She has rich teaching experience of 11 years.

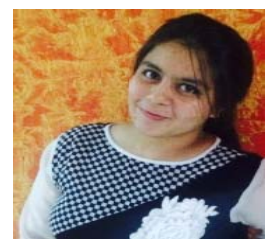

Amritjot Kaur Student Deptt.of C.S.E, SGGSWU, F.G.S, Punjab. She has done B. Tech from SGGSWU and Pursuing M. Tech from SGGSWU. 